\title{
Pancreatic Somatostatin-Producing Neuroendocrine Tumor
}

National Cancer Institute

\section{Source}

National Cancer Institute. Pancreatic Somatostatin-Producing Neuroendocrine Tumor. NCl Thesaurus. Code C8006.

A usually malignant, somatostatin producing neuroendocrine tumor, arising from the delta cells of the pancreas. It may or may not be associated with inappropriate secretion of somatostatin and an associated clinical syndrome. 